PROCEEDINGS OF THE

AMERICAN MATHEMATICAL SOCIETY

Volume 135, Number 7, July 2007, Pages 2263-2271

S 0002-9939(07)08728-X

Article electronically published on March 2, 2007

\title{
SPACES WITH MANY AFFINE FUNCTIONS
}

\author{
PETRA HITZELBERGER AND ALEXANDER LYTCHAK
}

(Communicated by Alexander N. Dranishnikov)

\begin{abstract}
We describe all metric spaces that have sufficiently many affine functions. As an application we obtain a metric characterization of linearconvex subsets of Banach spaces.
\end{abstract}

\section{INTRODUCTION}

Given a geodesic metric space $X$ the existence of a non-constant affine function on $X$ seems to impose strong restriction on the geometry of the space. Here a function $f: X \rightarrow \mathbb{R}$ is called affine if its restriction on each arclength parametrized geodesic $\gamma$ in $X$ satisfies $f(\gamma(t))=a t+b$ for some numbers $a, b$ that may depend on $\gamma$. For instance, if $X$ is a complete Riemannian manifold, such a function happens to exist if and only if $X$ has a non-trivial Euclidean de Rham factor (【nn82]) and the same is true in some more general spaces (Mas02, $\mathrm{AB} 05$ ]).

However, it is not clear what happens in the general situation. The expectation is that the existence of such a function forces the space $X$ to look like a Banach space in some "direction" defined by the affine function; compare the main result in LS04 and examples there. Thus it seems natural to expect that if $X$ has sufficiently many affine functions it should look very similar to some Banach space. Our result confirms this expectation:

Theorem 1.1. Let $X$ be a geodesic metric space. Affine functions on $X$ separate points of $X$ if and only if $X$ is isometric to a convex subset of a normed vector space with a strictly convex norm.

Here we say that affine functions separate points of $X$ if for each pair of points $x, \bar{x} \in X$ there is an affine function $f: X \rightarrow \mathbb{R}$ with $f(x) \neq f(\bar{x})$.

It is possible to look on our theorem from another point of view. In order to do this we define a constant speed geodesic in a metric space to be a curve that has a constant speed and is globally minimizing between its endpoints. A map $F: X \rightarrow Y$ between geodesic metric spaces is called affine if it sends each constant speed geodesic in $X$ to a constant speed geodesic in $Y$. We say that $F$ is an affine equivalence if $F$ is bijective and $F$ and $F^{-1}$ are affine.

It is a natural question as to which extent constant speed geodesics determine the metric on a space, i.e. given a geodesic metric space $X$ what are all metric spaces $Y$ that are affinely equivalent to the space $X$ ? If $X$ and $Y$ are complete

Received by the editors December 1, 2005 and, in revised form, March 28, 2006.

2000 Mathematics Subject Classification. Primary 53C20.

Key words and phrases. Affine functions, Banach spaces, geodesic mappings.

(C)2007 American Mathematical Society

Reverts to public domain 28 years from publication 
Riemannian manifolds the answer to this question has been known for a long time (Inn82]) and says that $Y$ has a de Rham decomposition that consists of direct factors of $X$ stretched by constants.

Remark 1.2. If (still in the realm of Riemannian geometry) one relaxes the condition of affine equivalence to that of projective equivalence, i.e. if one only requires the geodesics of $X$ and $Y$ to coincide as subsets (unregarding the parametrization), the question becomes much deeper. In fact it is a very old problem to which many partial answers are known; see, for instance, [Mat03] and the literature there.

If (while looking for affinely equivalent spaces) we still ask $X$ to be a Riemannian manifold but allow $Y$ to be arbitrary, then the answer of $[\mathrm{Inn} 82$ must change. For example, the Euclidean space is affine equivalent to each normed vector space of the same dimension with a strictly convex norm. As a consequence of our result we see that nothing more can happen if $X$ is Euclidean. Namely the notion of an affine function does not change under affine equivalence; hence from Theorem 1.1 we derive:

Corollary 1.3. Let $C$ be a convex subset of a Euclidean space $\mathbb{R}^{n}$. If $Y$ is a geodesic metric space that is affinely equivalent to $C$, then one can equip $\mathbb{R}^{n}$ with a strictly convex norm such that $C$ in this new norm is isometric to $Y$.

Remark 1.4. In Oht03 it is shown that if $X$ is a Riemannian manifold and $Y$ is affine equivalent to $X$, then the metric on $Y$ is defined by a continuous Finsler metric. We think that our arguments can help to describe the possible Finsler metric precisely.

The restriction to strictly convex norms in Theorem 1.1 seems artificial. However, one should be careful when trying to drop this restriction. In fact each metric space has an isometric embedding in some (non-strictly convex) Banach space. On the other hand $\mathbb{R}^{2}$ with the maximum norm $\|(x, y)\|=\max \{|x|,|y|\}$ has too many geodesics to admit an affine function in the sense of the definition above. To avoid these difficulties one has to distinguish good (i.e. linear) geodesics from bad ones, thus we need the notion of a bicombing.

Let $X$ be a metric space. A bicombing $\Gamma$ on $X$ is an assignment to each pair $x, y$ of points in $X$ a geodesic $\gamma_{x y}$ connecting $x$ and $y$, such that $\gamma_{y x}$ is the geodesic $\gamma_{x y}$ with the reverse orientation. Moreover, we require that for a point $m$ on a geodesic $\gamma_{x y}$ the geodesic $\gamma_{m y}$ is part of the geodesic $\gamma_{x y}$. We do not require that the geodesics $\gamma_{x y}$ depend continuously on $x$ and $y$.

We say that a map $F: X \rightarrow Y$ between two spaces with bicombings denoted by the same letter $\Gamma$ is $\Gamma$-affine if for all $x, \bar{x} \in X$ there are some numbers $a, b$ such that $f\left(\gamma_{x \bar{x}}(t)\right)=\gamma_{f(x) f(\bar{x})}(a t+b)$ holds.

Example 1.5. If $X$ is a uniquely geodesic metric space, i.e. if between every two points of $X$ there is precisely one geodesic, then we have a unique bicombing $\Gamma$ on $X$, and a $\Gamma$-affine function on $X$ is just an affine function in the sense of the old definition.

Example 1.6. Each normed vector space has a natural bicombing that assigns to two points the linear interval between them $\left(\gamma_{x y}(t)=x+t \frac{y-x}{\| y-x||}\right)$. All normed vector spaces will be considered with this bicombing $\Gamma$ in the sequel. With this definition each linear map between two normed vector spaces is a $\Gamma$-affine map. On 
the other hand each $\Gamma$-affine map $F: V \rightarrow W$ between normed vector spaces has the form $F(v)=F(0)+A(v)$ for some linear map $A$, i.e. $F$ is affine in the usual sense of linear algebra.

Using this notion we can now extend Theorem 1.1

Theorem 1.7. Let $X$ be a space with a bicombing $\Gamma$. Then the following are equivalent:

(1) There is a Banach space $B$ and an injective isometric $\Gamma$-affine map $f$ : $X \rightarrow B$.

(2) The $\Gamma$-affine functions separate the points of $X$.

(3) There is a Banach space $B$ and an injective $\Gamma$-affine map $f: X \rightarrow B$.

The equivalence between (2) and (3) can be reformulated as a metric characterization of linearly convex subsets of Banach spaces:

Corollary 1.8. Let $X$ be a metric space. Then $X$ is isometric to a linearly convex subset of some Banach space if and only if $X$ admits a bicombing $\Gamma$ such that the $\Gamma$-affine functions separate points of $X$.

A short comment on the regularity of affine functions. It is well known that an affine function on a Riemannian manifold is smooth. However, in general spaces affine functions do not need to be even continuous, for instance, there are many non-continuous linear functions on Hilbert spaces. In our theorem we did not make any restrictions on the affine functions, but a combination of Theorem 1.7 and the Hahn-Banach theorem immediatly shows that for each geodesic metric space $X$ Lipschitz continuous affine function separate points of $X$ if arbitrary affine functions separate points of $X$.

The paper is organized as follows. In Section 3 we show that Theorem 1.7 implies Theorem 1.1 and prove the straightforward implications $(1) \rightarrow(2) \rightarrow(3)$ in Theorem 1.7. In Section 4 we reduce the proof of the implication $(3) \rightarrow(1)$ to the case where $X$ is an open convex subset of $\mathbb{R}^{n}$ with some Finsler metric on it. Finally in Section 5 we first discuss variation formulas in this Finsler metric and then prove that the Finsler structure is constant.

We would like to express our gratitude to Werner Ballmann for his encouragement and support. We are grateful to Linus Kramer for helpful comments.

\section{Preliminaries}

2.1. Vector spaces. Linear intervals in a vector space $V$ are curves $\gamma:[0,1] \rightarrow V$ of the form $\gamma(t)=t v+(1-t) w$ for some $v, w \in V$. A subset $C$ of $V$ is called linearly convex if it contains each linear interval $\gamma$ as above for all $v, w \in C$. Let $C$ be a linearly convex subset of $V$ that contains the origin 0 . Then the linear hull $H(C)$ of $C$ (i.e. the smallest linear subset of $V$ that contains $C$ ) is the set of all points $x$ that can be represented as $x=\lambda(v-w)$ for some $\lambda>0$ and some $v, w \in C$. Let $C \subset V$ be a linearly convex subset of $V$ that contains the origin and assume that $V$ coincides with the linear hull $H(C)$ of $C$. By the dimension of $C$ we denote the dimension of the vector space $V=H(C)$. If the dimension of $C$ is finite, it is well known that the set $O$ of inner points of $C$ (with respect to the usual Euclidean topology of $H(C))$ is convex, non-empty and that the closure $\bar{O}$ of $O$ in $V$ contains C. 
2.2. Metric spaces. By $d$ we will denote distances in metric spaces. A geodesic respectively a ray in a metric space $X$ will denote an isometric embedding $\gamma: I \rightarrow X$ of an interval respectively of a half-line into $X$. Note that geodesics (if not otherwise stated) are parametrized by the arclength.

A metric space is called geodesic if each pair of its points is connected by a geodesic. It is called uniquely geodesic if this connecting geodesic is unique.

Given a ray $\gamma$ in a metric space $(X, d)$ the Busemann function of $\gamma$ is defined by $b_{\gamma}(x):=\lim _{t \rightarrow \infty}(d(\gamma(t), x)-d(\gamma(0), \gamma(t)))$. The Busemann function exists and is a 1-Lipschitz function on $X$. Moreover $b_{\gamma}(x) \leq d(\gamma(t), x)-d(\gamma(0), \gamma(t))$ for all $t \geq 0$.

2.3. Basics on normed vector spaces. Let $(V,\|\cdot\|)$ be a vector space with a norm and denote by $d$ the induced metric on $V$. The norm is called strictly convex if for all linear independent $v, w \in V$ one has $\|v+w\|<\|v\|+\|w\|$. The norm $\|\cdot\|$ is strictly convex if and only if $(V, d)$ is uniquely geodesic.

For each non-zero vector $h$ in $V$ we denote by $\gamma^{h}$ the ray $\gamma^{h}(t)=t \frac{h}{\|h\|}$. The Busemann functions $b_{h}:=b_{\gamma^{h}}$ of such linear rays have the following properties, that are direct consequences of the definition:

(1) $b_{h}(v)=\lim _{t \rightarrow \infty}(\|t h-v\|-\|t h\|)=\lim _{t \rightarrow 0^{+}}\left(\frac{\|h-t v\|-\|h\|}{t}\right)$;

(2) $b_{h}(t v)=t b_{h}(v)$, for all $t \geq 0$;

(3) $b_{h}\left(v_{1}+v_{2}\right) \leq b_{h}\left(v_{1}\right)+b_{h}\left(v_{2}\right)$;

(4) $b_{h}(v)=b_{-h}(-v)$.

We see that the Busemann function $b_{h}$ is linear if and only if the equality $b_{h}(v)=$ $-b_{h}(-v)$ holds for all $v \in V$. However, this equality is equivalent to the following well known one:

Definition 2.1. A point $h \in V$ is called smooth in the norm $\|\cdot\|$ if the following holds for all $v \in V$ :

$$
\lim _{t \rightarrow 0^{+}} \frac{\|h-t v\|-\|h\|}{t}=-\lim _{t \rightarrow 0^{+}} \frac{\|h+t v\|-\|h\|}{t} .
$$

Thus the Busemann function $b_{h}$ is linear if and only if $h$ is a smooth vector in the norm $\|\cdot\|$. If $V$ is a finite dimensional vector space, then a vector $h$ is smooth in the norm $\|\cdot\|$ if and only if the Lipschitz map $\|\cdot\|: \mathbb{R}^{n} \rightarrow \mathbb{R}$ has a linear differential at the point $h$. Due to the theorem of Rademacher we know that in a finite dimensional normed vector space $V$ almost each vector is smooth with respect to the norm.

\section{EASY IMPLICATIONS}

3.1. Strictly convex case. First we are going to derive Theorem 1.1 from Theorem 1.7

Let $X$ be a space on which affine functions separate the points. Let $x, \bar{x}$ be two arbitrary points in $X$. For each geodesic $\gamma$ from $x$ to $\bar{x}$ each affine function $f$ has the value $\frac{f(x)+f(\bar{x})}{2}$ on the midpoint $m$ of $\gamma$. Thus all affine functions have the same value on the midpoint of each geodesic between $x$ and $\bar{x}$. From the separation assumption we deduce that $X$ is uniquely geodesic.

By Theorem 1.7 we know that $X$ is isometric to a linearly convex subset of a Banach space $B$. We replace $X$ by this subset and may assume that it contains the origin 0. Denote by $V$ its linear hull in $B$ with the norm induced from the ambient 
Banach space $B$. Each element $v$ of $V$ has the form $\lambda(x-\bar{x})$ for some $\lambda \geq 0$ and some $x, \bar{x} \in X$.

We claim that $V$ is strictly convex. Assume the contrary and find linearly independent $v, w \in V$ with $\|v+w\|=\|v\|+\|w\|$. We can find a finite dimensional linearly convex subset $\tilde{X}$ of $X$ that contains 0 and whose linear hull $\tilde{V}$ contains $v$ and $w$. Due to finite dimensionality $\tilde{X}$ contains an open ball in $\tilde{V}$, and since $\tilde{V}$ is not strictly convex, the subset $\tilde{X}$ is not uniquely geodesic. But $\tilde{X}$ is a convex subset of a uniquely geodesic space $X$, and we arrive at a contradiction.

The other direction is clear since linear functions are affine on a strictly convex normed vector space and separate its points (compare the next subsection).

3.2. Linear algebra. We prove $(1) \rightarrow(2)$ of Theorem 1.7 here. Thus let $(V,\|\cdot\|)$ be a normed vector space. Observe that each linear function on $V$ is $\Gamma$-affine with respect to the natural bicombing. Since linear functions separate points of $V$ (by linear algebra, you may also apply the Hahn-Banach theorem), we see that $\Gamma$-affine functions separate points of $V$. We deduce that $\Gamma$-affine functions also separate points on each space $X$ that admits an injective $\Gamma$-affine map $i: X \rightarrow V$.

3.3. Evaluation. Assume now (2) of Theorem 1.7. Consider the vector space $\Gamma A f f(X)$ of all $\Gamma$-affine functions on $X$ and denote by $V$ its (algebraic) dual space $(\Gamma A f f(X))^{*}$. Define the natural evaluation map $E: X \rightarrow V$ by $E(x)(f)=f(x)$ (compare [LS04]). The map is well defined, and by the separation property it is injective.

We claim that $E$ sends each $\Gamma$-geodesic onto a linearly reparametrized linear interval.

In fact, let a geodesic $\gamma_{x y}$ be given and reparametrize it to a constant speed geodesic $\bar{\gamma}_{x y}:[0,1] \rightarrow X$ such that $\bar{\gamma}_{x y}(0)=x, \bar{\gamma}_{x y}(1)=y$. For each $t \in[0,1]$ and each affine function $f: X \rightarrow \mathbb{R}$ we see $f\left(\bar{\gamma}_{x y}(t)\right)=(1-t) f(x)+t f(y)$. Hence for our map $E$ we deduce $E\left(\bar{\gamma}_{x y}(t)\right)=(1-t) E(x)+t E(y)$.

Now we equip $V$ with an arbitrary norm. The linear intervals become linearly reparametrized $\Gamma$-geodesics for the natural bicombing $\Gamma$ on $V$. Hence the map $E: X \rightarrow V$ becomes $\Gamma$-affine. Taking $B$ to be the completion of $V$ we obtain (3) of Theorem 1.7 .

\section{REDUCTION}

In this section we reduce the implication $(3) \rightarrow(1)$ of Theorem 1.7 to the case where $X$ is a Finsler manifold.

4.1. Reformulation. Let $X$ be a space with a bicombing $\Gamma$ that admits an injective $\Gamma$-affine map $i: X \rightarrow B$ into a Banach space $B$. We forget the norm on $B$ and identify $X$ with its image $C$. We may assume that 0 is contained in $C$ and replace $B$ by the linear hull $V$ of $C$ in $B$.

We have to prove that we can define a norm on $V$ such that the induced metric on $C$ coincides with the given one. Thus the implication we are looking for is implied by the following:

Lemma 4.1. Let $V$ be a vector space, and $C$ a linear convex subset of $V$ that contains the origin 0 . Let $d$ be a metric on $C$ such that the linear intervals contained in $C$ are constant speed geodesics with respect to the metric $d$. Then there is a norm $\|\cdot\|$ on $V$ such that the induced metric on $C$ coincides with $d$. 
4.2. Reduction to finite dimension. Since norms defined on linear subspaces can be extended to norms on the whole space it is enough to prove that such a norm exists on the linear hull $H(C) \subset V$. Each element $v \in H(C)$ has the form $v=\lambda(x-\bar{x})$ for some $x, \bar{x} \in C$ and some $\lambda \geq 0$. Thus the norm we are looking for is unique, if it exists, and must be given by $\|v\|=\lambda d(x, \bar{x})$, where $v$ has a presentation as above.

Thus we need to prove that this is a well defined quantity (i.e. independent of the representation of $v$ ) and that it defines a norm on $H(C)$.

Observe that each linearly convex subset $\bar{C}$ of $C$ that contains 0 and is considered with the metric induced by $d$ also satisfies the assumptions of Lemma 4.1. We deduce that it is enough to prove Lemma 4.1 in the case where the dimension of $C$ is finite. For instance, in order to see that $\|\cdot\|$ is well defined take a point $v \in H(C)$ that has two different presentations $v=\lambda(x-\bar{x})$ and $v=\lambda_{1}\left(x_{1}-\bar{x}_{1}\right)$. Consider the linear convex hull $\bar{C}$ of the five points $x, \bar{x}, x_{1}, \bar{x}_{1}, 0$. Assuming that Lemma 4.1 is true for the finite dimensional $\bar{C}$ we deduce that $\lambda d(x, \bar{x})=\lambda_{1} d\left(x_{1}, \bar{x}_{1}\right)$, hence $\|\cdot\|$ is well defined. The fact that $\|\cdot\|$ is a norm is shown in the same way.

Thus it is enough to prove Lemma4.1 in the case where $C$ has a finite dimension.

4.3. Reduction to open subsets. Let $C$ be as in Lemma 4.1 and assume that the dimension of $C$ is finite. Replacing $V$ by the linear hull $H(C)$ we may assume that $V=\mathbb{R}^{n}$. If Lemma 4.1 is true for the set $O$ of inner points of $C$, then by continuity it is also true for the closure $\bar{O}$ of $O$ in $\mathbb{R}^{n}$ and therefore it is also true for $C \subset \bar{O}$.

Thus it is enough to prove Lemma 4.1 in the case where $V=\mathbb{R}^{n}$ and where $C$ is a convex open subset of $\mathbb{R}^{n}$.

4.4. Reduction to a Finsler metric. Assume now that in Lemma 4.1 we have $V=\mathbb{R}^{n}$ and that $C$ is open. We claim that the metric $d$ on $C$ is defined by a continuous Finsler structure on $C$.

In fact it is more or less a special case of Theorem B in [Oht03. We shortly recall the arguments for the convenience of the reader, since the assumptions in Oht03. are slightly different. First of all one defines for each $x \in C$ and each $v \in \mathbb{R}^{n}$ the quantity $|v|_{x}$ as $d(x, x+\epsilon v) / \epsilon$, for a sufficiently small positive real number $\epsilon$. Since $\gamma(t)=x+t v$ is a constant speed curve on the whole interval that is contained in the open set $C$, we deduce that the definition of $|v|_{x}$ does not depend on the choice of $\epsilon$, and in fact it is just the speed of the curve $\gamma$.

Moreover, we immediately deduce that $|\lambda v|_{x}=|\lambda| \cdot|v|_{x}$. Since $d$ is a metric we have $|v|_{x} \geq 0$ with equality if and only if $v=0$.

We need to assure that the metric space $(C, d)$ is locally compact, i.e. that the identity $i d:\left(C, d_{E u c l}\right) \rightarrow(C, d)$ is continuous. This follows directly, as soon as one knows that for given $x \in C$ the function $|\cdot|_{x}$ is bounded on compact subsets of $\mathbb{R}^{n}$ (or equivalently on the unit ball). The last claim is the content of Proposition 2.1 in Oht03. Now fix a small $\epsilon$ and take a convergent sequence $x_{i} \rightarrow x$ and a convergent sequence $v_{i} \rightarrow v$. We observe that $x_{i}+\epsilon v_{i}$ converge to $x+\epsilon v$, hence by definition $\left|v_{i}\right|_{x_{i}}$ must converge to $|v|_{x}$. Therefore the function $|v|_{x}$ is continuous in $v$ and $x$. From this we can derive that $|\cdot|_{x}$ is a norm. Namely, we have $d(x, x+\epsilon(v+w)) \leq d(x, x+\epsilon v)+d(x+\epsilon v, x+\epsilon v+\epsilon w)=\epsilon|v|_{x}+\epsilon|w|_{x+\epsilon v}$. Dividing by $\epsilon$ we get in the limit $|v+w|_{x} \leq|v|_{x}+|w|_{x}$. 
Thus $|\cdot|_{x}$ is a continuous Finsler structure and defines a metric $\tilde{d}$ on $C$. The conclusion that $\tilde{d}$ coincides with $d$ is a direct consequence of the fact that linear intervals have the same length with respect to the metric $d$ and $\tilde{d}$.

Thus we have shown that the metric $d$ is given by a continuous Finsler structure $|\cdot|_{x}$ on $C$.

\section{FinAL STEP}

We are left with the following problem. Let $O \subset \mathbb{R}^{n}$ be an open linear convex subset. Let $|\cdot|_{x}$ be a continuous Finsler structure on $O$, such that for the induced metric $d$ the linear intervals are constant speed geodesics. We need to show that there is a norm $\|\cdot\|$ on $\mathbb{R}^{n}$ such that the metric $\tilde{d}$ on $O$ induced by this norm coincides with our metric $d$. This conclusion follows as soon as we know that $|\cdot|_{x}=|\cdot|_{y}$ holds for all $x, y \in O$. The rest of this section is devoted to the proof of the last statement.

First of all linear intervals have constant speed, hence for each $x \in O$, each $v \in \mathbb{R}^{n}$ and each $t \in \mathbb{R}$ such that $x+t v$ is still in $O$ we have $|v|_{x}=|v|_{x+t v}$. Since linear intervals are geodesics we conclude that $d(x, x+t v)=|t v|_{x}$, i.e. for all $x, y \in O$ we have $d(x, y)=|x-y|_{x}$. (To avoid confusion: what we need is the much stronger statement $d(y, z)=|y-z|_{x}$ for all $x, y, z \in O$ !)

Let us fix an arbitrary $v \in \mathbb{R}^{n}$. It is enough to show that $|h|_{x}=|h|_{x+v}$ for almost all $h \in \mathbb{R}^{n}$ and all $x, x+v \in O$. Here and below we consider $\mathbb{R}^{n}, O$ the tangent bundle $T O=O \times \mathbb{R}^{n}$ and linear intervals equipped with natural Lebesgue measures. By abuse of notation we will say that $h \in \mathbb{R}^{n}$ is smooth at a point $x \in O$ if $h$ is a smooth vector of the norm $|\cdot|_{x}$ (compare Definition 2.1). For each $x \in O$ almost all $h \in \mathbb{R}^{n}$ are smooth at $x$. Applying Fubini's theorem twice we find a set $S$ of full measure in $\mathbb{R}^{n}$ with the following property. For each vector $h \in S$ the set of all $x \in O$ at which $h$ is smooth is of full measure in $O$.

It is enough to show that for each $h \in S$ the equality $|h|_{x}=|h|_{x+v}$ holds, for all $x, x+v \in O$. Let us fix a vector $h \in S$. Another application of Fubini's theorem tells us that for almost all $x \in O$ the vector $h$ is smooth at almost each point of the linear interval $\eta(t)=x+t v$ (contained in $O$ ). By continuity of the Finsler structure it is enough to show that $f(t)=|h|_{x+t v}$ does not depend on $t$ if $h$ is smooth at almost each point of $\eta$. The question is local in $t$, hence it is enough to show that $f(t)$ is constant for all small $t$. We may assume (replacing $h$ by its multiple) that $x+h$ is contained in $O$. Then $f(t)=d(x+t v, x+t v+h)$, in particular, the function $f$ is locally Lipschitz. Hence it is enough to show that $f^{\prime}(t)=0$ for almost all $t$. Therefore our result is a consequence of the following lemma.

Lemma 5.1. Let $x$ be a point in $O, h, v \in \mathbb{R}^{n}$. Assume that $h$ is a smooth vector in the norm $|\cdot|_{x}$. Then for the function $f(t)=|h|_{x+t v}$ we have $f^{\prime}(0)=0$.

Proof. Replacing $h$ by its multiple we may assume that $y:=x+h$ is contained in $O$. Our claim is $|h|_{x+t v}=|h|_{x}+o(t)$, where $o(t)$ is some function with $\lim _{t \rightarrow 0} \frac{o(t)}{t}=0$. Since $|h|_{x+t v}=d(x+t v, y+t v)$ we just have to find the right first variation formulas in the Finsler manifold $O$. We refer to Section 9 in Lyt04 for a general discussion of first variation formulas. We need some notation. For a vector $w \in \mathbb{R}^{n}$ and a point $z \in O$ we will denote by $b_{w}^{z}: \mathbb{R}^{n} \rightarrow \mathbb{R}$ the Busemann function of the direction $w$ in the norm $|\cdot|_{z}$, i.e. $b_{w}^{z}(v)=\lim _{t \rightarrow \infty}\left(|t w-v|_{z}-|t w|_{z}\right)$. We refer to Subsection 2.3 for basic properties of such functions. 
First we are going to show that $b_{h}^{x}=b_{h}^{y}$. In order to do so we have to study the first derivative of the function $\tilde{f}(t)=d(y, x+t v)$. Recall that $|h|_{x}=|h|_{y}$.

Due to $d(y, x+t v)=|y-x-t v|_{y}=|h-t v|_{y}$ and properties of the Busemann functions (see Subsection 2.3) we have

$$
d(y, x+t v)=|h|_{y}+t b_{h}^{y}(v)+o(t) .
$$

On the other hand we can estimate $d(y, x+t v)$ by working in a small neighborhood of $x$. Fix $s \gg 1$. For $0<t \ll \frac{1}{s}$ we have $d(y, x+t v) \leq d(y, x+t s h)+d(x+t s h, x+t v)$. Since the metric on $O$ is given by a continuous Finsler structure, we have

$$
d(x+t s h, x+t v)=t|s h-v|_{x}+o(t)
$$

(i.e. $\left(\mathbb{R}^{n},|\cdot|_{x}\right)$ is the tangent space of $O$ at $x$ in the sense of metric geometry, Lyt04). We obtain $d(y, x+t v) \leq|h|_{x}(1-t s)+t|s h-v|_{x}+o(t)$. If $s$ is large enough $|s h-v|_{x}$ is very close to $s|h|_{x}+b_{h}^{x}(v)$. Letting $s$ go to infinity we conclude that

$$
d(y, x+t v) \leq|h|_{x}+t b_{h}^{x}(v)+o(t) \quad \text { for } t>0 .
$$

Consider $d(y, x+t v)+d(x+t v, x-t s h) \geq d(y, x-t s h)=|h|_{x}(1+t s)$ for a fixed large $s$ and let $t$ go to $0^{+}$. Then as above we obtain

$$
d(x+t v, y) \geq|h|_{x}-t b_{-h}^{x}(v)+o(t) \quad \text { for } t>0 .
$$

By our assumption vector $h$ is smooth at $x$, thus $b_{-h}^{x}(v)=-b_{h}^{x}(v)$. Combining $(1),(2),(3)$ above we deduce that $b_{h}^{x}(v)=b_{h}^{y}(v)$. Since the above calculations work for all $v \in \mathbb{R}^{n}$ we get $b_{h}^{x}=b_{h}^{y}$. In particular, $h$ is also smooth in $|\cdot|_{y}$ and we have $b_{-h}^{x}=b_{-h}^{y}$. Now we can finish the proof of Lemma 5.1. As in the proof of (2) and (3) above we take a large $s$ and estimate the distance $d(x+t v, y+t v)$ from above (for all small $0<t \ll \frac{1}{s}$ ) by

$$
\begin{aligned}
d(x+t v, y+t v) \leq & d(x+t v, x+t s h)+d(x+t s h, y-t s h) \\
& +d(y-t s h, y+t v) .
\end{aligned}
$$

Due to the continuity of the Finsler structure we have

$$
\begin{gathered}
d(x+t v, x+t s h)=t|v-s h|_{x}+o(t) \\
\text { and } \quad d(y-t s h, y+t v)=t|v+s h|_{y}+o(t) .
\end{gathered}
$$

Thus we conclude

$$
d(x+t v, y+t v) \leq t|v-s h|_{x}+|h|(1-2 t s)+t|v+s h|_{y}+o(t) .
$$

If $s$ goes to infinity, then $|v-s h|_{x}-s|h|_{x}$ converges to $b_{h}^{x}(v)$ and $|v+s h|_{y}-s|h|_{y}$ converges to $b_{-h}^{y}(v)$. Thus we arrive at:

$$
d(x+t v, y+t v) \leq|h|+t b_{h}^{x}(v)+t b_{-h}^{y}(v)+o(t) .
$$

Now we take a large $s$ and estimate $d(x+t v, y+t v)$ from below by

$$
\begin{aligned}
d(x+t v, y+t v) \geq & d(x-t s h, y+t s h)-d(x-t s h, x+t v) \\
& -d(y+t v, y+t s h) .
\end{aligned}
$$

As above we derive from it

$$
d(x+t v, y+t v) \geq|h|-t b_{-h}^{x}(v)-t b_{h}^{y}(v)+o(t) .
$$

Since we know that $b_{h}^{x}=b_{h}^{y}=-b_{-h}^{y}=-b_{-h}^{x}$ we can combine (4) and (5) and obtain $d(x+t v, y+t v)=d(x, y)+o(t)$. Thus we have shown $f^{\prime}(0)=0$. 
This finishes the proof of Theorem 1.7

\section{REFERENCES}

[AB05] S. Alexander and R. Bishop, A cone splitting theorem for Alexandrov spaces, Pacific J. Math. 218 (2005).

[Inn82] N. Innami, Splitting theorems of Riemannian manifolds, Compositio Math. 47 (1982), $237-247$.

[LS04] A. Lytchak and V. Schroeder, Affine functions on $(C A T(\kappa))$ spaces, Math. Z. 255 (2007), 231-244.

[Lyt04] A. Lytchak, Differentiation in metric spaces, Algebra i Analiz 16 (2004), no. 6, 128-161. MR2117451

[Mas02] Yukihiro Mashiko, A splitting theorem for Alexandrov spaces, Pacific J. Math. 204 (2002), no. 2, 445-458. MR1907900 (2003b:53044)

[Mat03] V. Matveev, Hyperbolic manifolds are geodesically rigid, Invent. math 151 (2003), 579609.

[Oht03] S. Ohta, Totally geodesic maps into metric spaces, Math. Z. 244 (2003), no. 1, 47-65. MR:1981876 (2004k:53059)

Mathematisches Institut, Fachbereich Matho \& Info, Uni Müenster, Einsteinstrasse 62, 48149 Muenster, Germany

E-mail address: hitzelberger@uni-muenster.de

Mathematisches Institut, Universität Bonn, Beringstr. 1, 53115 Bonn, Germany

E-mail address: lytchak@math.uni-bonn.de 